\title{
A Novel Canine Model for Prostate Cancer
}

\author{
Jill M. Keller, ${ }^{1}$ George R. Schade, ${ }^{1}$ Kimberly Ives, ${ }^{1}$ Xu Cheng, ${ }^{1}$ Thomas J. Rosol, ${ }^{2}$ \\ Morand Piert, ${ }^{3}$ Javed Siddiqui, ${ }^{4}$ William W. Roberts, ${ }^{1}$ and Evan T. Keller ${ }^{1 *}$ \\ 'Department of Urology, University of Michigan, Ann Arbor, Michigan \\ ${ }^{2}$ Department of Veterinary Biosciences, The Ohio State University, Columbus, Ohio \\ ${ }^{3}$ Department of Radiology, University of Michigan, Ann Arbor, Michigan \\ ${ }^{4}$ Center for Translational Pathology, University of Michigan, Ann Arbor, Michigan
}

\begin{abstract}
BACKGROUND. No existing animal model fully recapitulates all features of human prostate cancer. The dog is the only large mammal, besides humans, that commonly develops spontaneous prostate cancer. Canine prostate cancer features many similarities with its human counterpart. We sought to develop a canine model of prostate cancer that would more fully represent the features of human prostate cancer than existing models.

METHODS. The Ace- 1 canine prostate cancer cell line was injected transabdominally under transrectal ultrasound (TRUS) guidance into the prostates of immunosuppressed, intact, adult male dogs. Tumor progression was monitored by TRUS imaging. Some dogs were subjected to positron emission tomography (PET) for tumor detection. Time of euthanasia was determined based on tumor size, impingement on urethra, and general well-being. Euthanasia was followed by necropsy and histopathology.

RESULTS. Ace-1 tumor cells grew robustly in every dog injected. Tumors grew in subcapsular and parenchymal regions of the prostate. Tumor tissue could be identified using PET. Histological findings were similar to those observed in human prostate cancer. Metastases to lungs and lymph nodes were detected, predominantly in dogs with intraprostatic tumors.

CONCLUSIONS. We have established a minimally invasive dog model of prostate cancer. This model may be valuable for studying prostate cancer progression and distant metastasis. Prostate 73: 952-959, 2013. (C) 2013 Wiley Periodicals, Inc.
\end{abstract}

KEY WORDS: animal model; dog; prostate cancer; metastasis

\section{INTRODUCTION}

Prostate cancer is the most frequently diagnosed malignancy and the third leading cause of cancer-related death in men in developed countries [1]. Animal models of prostate cancer, including a wide variety of transgenic, knockout, and xenograft mouse models, have proven extremely valuable in expanding our knowledge of this disease [2]. However, prostate cancer is a complex, multifactorial disease process, and despite the advances provided by animal models, no existing animal model fully recapitulates all features of human prostate cancer [3]. Besides humans, the dog is the only other large mammal which commonly develops spontaneous prostate cancer [4]. Similar to humans, dogs develop benign prostatic hyperplasia $(\mathrm{BPH})$ [5], high-grade prostatic intraepithelial neoplasia, and invasive prostate cancer spontaneously with age $[4,6]$. The dog prostate also shares a variety of anatomic and functional similarities with humans [4]. In addition, the disease process in dogs with spontaneous prostate cancer is very similar to that of humans, including a tendency to metastasize to bone, a feature which mouse models of induced prostate cancer do not share [7]. We sought to develop a dog model of prostate cancer that would more fully represent the features of human prostate cancer than

Grant sponsor: University of Michigan Department of Urology Pilot project fund and National Institutes of Health grant number P01 CA093900.

*Correspondence to: Evan T. Keller, Department of Urology, University of Michigan, Rm. 5308 Cancer Center, Ann Arbor, MI 48109-5940. E-mail: etkeller@umich.edu

Received 17 September 2012; Accepted 18 December 2012 DOI 10.1002/pros. 22642

Published online 17 January 2013 in Wiley Online Library (wileyonlinelibrary.com). 
existing rodent models. Such a dog model could prove invaluable in pre-clinical studies of diagnostic and therapeutic regimens for both human and canine prostate cancer.

\section{MATERIALS AND METHODS}

\section{Cell Culture}

The Ace- 1 cell line is a spontaneously immortalized canine prostate cancer cell line derived from a spontaneous prostatic adenocarcinoma that forms osteoblastic bone metastases in nude mice after intracardiac injection [8-10]. Cells were maintained at $37^{\circ} \mathrm{C}$ and $5 \%$ $\mathrm{CO}_{2}$ in RPMI medium supplemented with $10 \%$ fetal bovine serum (FBS) and 1\% penicillin/streptomycin.

\section{Subjects}

After receiving approval from the university committee for animal use and care, 12 intact 5- to 6-yearold male beagles weighing 11-14 kg were obtained. Subjects were administered oral cyclosporine at a starting dose of $200 \mathrm{mg}$ daily (range of 14.6-17.4 mg/ $\mathrm{kg}$ ). Cyclosporine levels were monitored biweekly and cyclosporine administration was titrated to achieve therapeutic cyclosporine trough levels between 400 and $600 \mathrm{ng} / \mathrm{dl}$. Once this window was achieved subjects were maintained in the therapeutic window throughout the remainder of the study.

\section{Tumor Implantation}

Once serum cyclosporine concentrations were maintained in the therapeutic window for 1 week, dogs were anesthetized with subcutaneous acepromazine $(0.1 \mathrm{mg} / \mathrm{kg})$ and intravenous propofol $(2-8 \mathrm{mg} / \mathrm{kg})$ and the trachea was intubated. The dogs were prepped with a tap water enema with digital rectal disimpaction after intubation. Inhalational anesthesia (isoflurane 1-2\%) was maintained throughout the procedure. All subjects received intramuscular Penicillin G benzathine/procaine $(40,000 \mathrm{IU} / \mathrm{kg})$ prior to the procedure for prophylaxis. The lower abdomen was shaved before positioning each subject supine on the procedural table and the lower abdomen was then prepped with povidine iodine and draped in a sterile fashion. Transrectal ultrasound (TRUS) imaging was performed using a Logiq 6 ultrasound scanner (GE Healthcare, Waukesha, WI) with a model ERB probe positioned manually. The prostate volume was estimated using the ellipsoid estimation $(\mathrm{H} \times \mathrm{W} \times \mathrm{L} \times \pi / 6)$. A 22gauge spinal needle was introduced through the abdominal skin to the left of the penis and passed into the left lobe of the prostate obliquely (in order to lengthen the needle path) under TRUS guidance. Both sagittal and transverse plane imaging was used to verify placement of the needle into the central left lobe at least $1 \mathrm{~cm}$ from the urethra. Once in position, a cellular suspension of Ace- 1 cells was injected into the prostate. In the first two subjects, $0.5 \mathrm{cc}$ of a $1.2 \times 10^{8}$ cells $/ \mathrm{cc}$ suspension was injected into the left hemiprostate $\left(6 \times 10^{7}\right.$ cells $)$ at a single site. Subsequent subjects had $0.5 \mathrm{cc}$ of a $6 \times 10^{7}$ cells $/ \mathrm{cc}$ suspension of Ace- 1 cells $\left(3 \times 10^{7}\right.$ cells $)$ in medium injected into the prostate in $0.1 \mathrm{cc}$ increments (5 boluses total) with slight repositioning of the needle between boluses in an effort to improve central tumor uptake. In all subjects, injection of cells was associated with the appearance of hyperechoic foci in the prostatic parenchyma (Fig. 1). If the initial injection resulted in clear efflux of suspension into the urethra, the needle was repositioned more laterally prior to the next injection. For subjects $6-12$, the last aliquot of cells was followed with $0.2 \mathrm{cc}$ of $0.1 \%$ gelatin in saline to help create a "plug" in the prostatic capsule.

\section{Tumor Surveillance}

Following injection of tumor cells, subjects were monitored for signs of disseminated tumor with daily physical examination. All subjects underwent weekly TRUS under anesthesia, using the same anesthetic protocol as above, to examine tumor growth and changes in prostate volume using the ellipsoid method. Beginning 2 weeks post-injection weekly flexible cystourethroscopy with an 8.2 French flexible ureteroscope (Dur-8, Gyrus ACMI) was performed following TRUS under anesthesia to assess for intraurethral tumors.

\section{CirculatingTumor Cells (CTCs)}

The Circulating Tumor Cell (CTC) Assay (Veridex, LLC, Raritan, NJ) was performed on whole blood

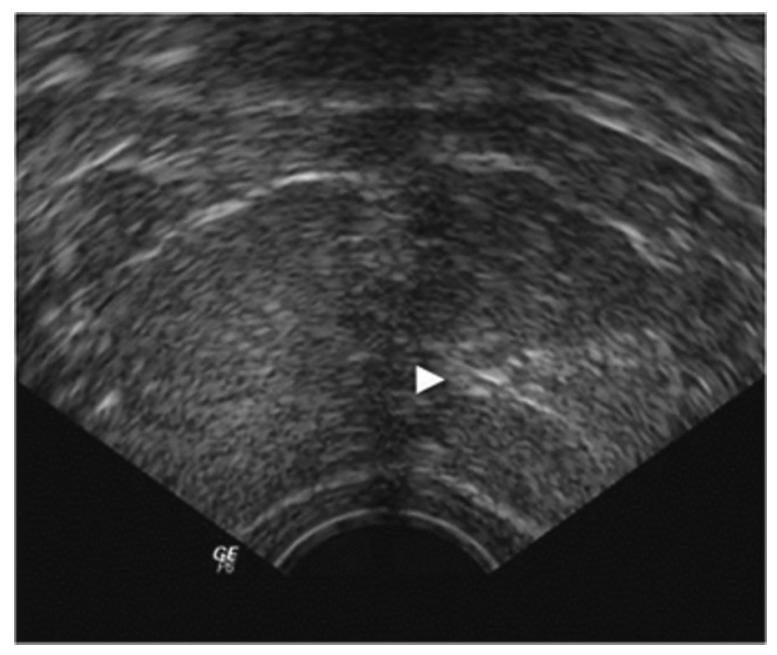

Fig. I. Orthotopic injection of Ace-I tumor cells into dog prostate under transrectal ultrasound guidance. Note the hyperechoic appearance of injection site (arrowhead) in left hemiprostate. 
samples collected from four dogs prior to euthanasia. This assay uses a ferrofluid-based capture reagent and immunofluorescent reagent to identify circulating tumor cells (CTCs). The ferrofluid reagent contains magnetic particles and antibodies that target the EpCAM antigen and immunomagnetically captures those cells which express EpCAM from other components within the blood sample. The fluorescent reagent which stains the captured cells includes antiCK-Phycoerythrin (PE, antibodies to cytokeratins 8, 18, and 19), DAPI and anti-CD45-Allophycocyanin (APC). Cell images are evaluated for shape, size, and staining pattern. The CTC Assay is reported out as the number of CTCs found in a $7.5 \mathrm{ml}$ sample of whole blood.

\section{Positron EmissionTomography (PET) and Data Analysis}

PET scan imaging was performed on two dogs. Following overnight fasting, both subjects were anesthetized with IV acepromazine/propofol followed by endotracheal intubation and maintenance on 1-3\% isoflurane as described previously. Anesthetized animals were positioned supine in a microPET P4 scanner (Concorde Microsystems, Inc., Knoxville, TN) centering the prostate in the field of view. The scanner table was extended to support the length of the dogs. Following a measured transmission scan, the first dog received a 60-min dynamic PET study of the pelvis immediately after intravenous injection of $4.1 \mathrm{mCi}$ of ${ }^{11} \mathrm{C}$-choline. The second dog received a $60-$ min pelvic scan after injection of $6.2 \mathrm{mCi}$ of ${ }^{11} \mathrm{C}$-methionine. Data were reconstructed using iterative ordered subset expectation maximization-maximum a posteriori (OSEM-MAP) yielding a reconstructed image resolution of approximately $1.4 \mathrm{~mm}$. Immediately after imaging, both animals were euthanized and specimens collected and processed as described elsewhere following complete decay for radioactivity.

\section{Euthanasia and Specimen Processing}

Subjects were euthanized with pentobarbital sodium $140-160 \mathrm{mg} / \mathrm{kg}$ IV 2-6 weeks after Ace-1 cell injection. Time of euthanasia was determined based on tumor size, impingement on urethra, and general subject well-being. At the time of euthanasia TRUS imaging was performed, followed by a suprapubic incision entering the peritoneum. The prostate and bladder were then surgically removed en bloc along with any extraprostatic tumor. A bilateral pelvic lymph node dissection was then conducted including the obturator and internal, external, and common iliac lymph nodes. If further retroperitoneal lymph nodes were enlarged they were harvested. Next, the adrenal glands and a portion of the liver were harvested followed by a thoracotomy and bilateral pneumonectomy with excision of any macroscopic or palpable lung nodules. Harvested tissues were fixed in formalin for 1 week, cut into 5-mm thick slabs, dehydrated in $25 \%$ ethanol, paraffin embedded, cut using a microtome in $5 \mu \mathrm{m}$ sections at $1 \mathrm{~mm}$ increments, mounted, and stained with hematoxylin and eosin for histologic assessment. All specimens were reviewed and interpreted by a veterinary pathologist (TJR).

\section{RESULTS}

Our initial procedure was to immunosuppress the dogs prior to tumor implantation to minimize rejection of the implanted cells by the host dogs. Cyclosporine is a commonly used immunosuppressant for tissue implantation; however, there is a wide variation in pharmacokinetics among individuals which requires close monitoring of cyclosporine levels to achieve appropriate immunosuppressive serum levels. We found that the final dose of cyclosporine needed to maintain target trough levels of 400-600 ng/dl varied widely among individuals and ranged from 12.1 to $40.0 \mathrm{mg} / \mathrm{kg} /$ day (Fig. 2). Stable cyclosporine target levels were achieved in approximately 2 weeks in most dogs.

Ace-1 tumor cells grew robustly in every dog injected. Following injection of cells, a hyperechoic area could be identified at the injection site on transrectal ultrasound images (Fig. 1). Injection of the first two dogs, which was performed as a single bolus, resulted in tumor establishment within 1 week in the subcapsular area of the prostate (Fig. 3). Tumors arising within

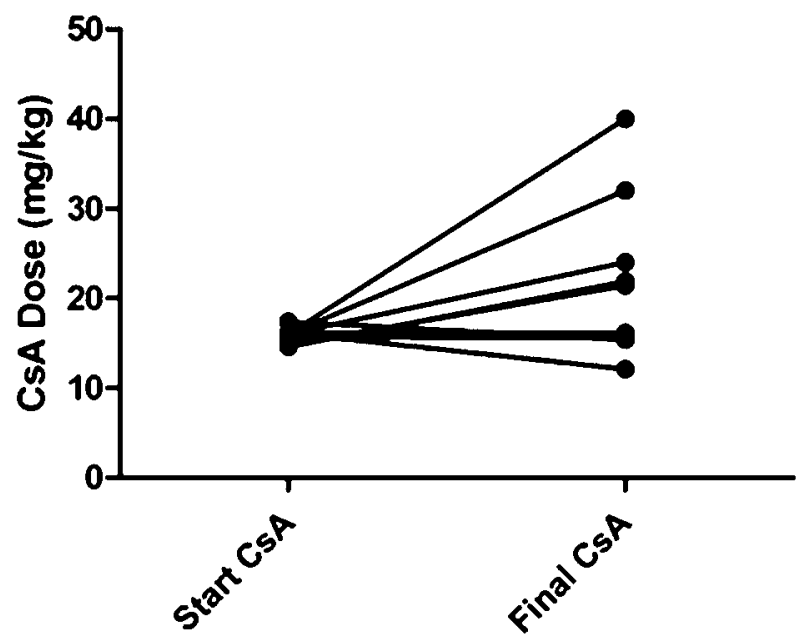

Fig. 2. Cyclosporine $(C s A)$ dosages $(n=12)$. Each dog initially received $200 \mathrm{mg}$ CsA daily, resulting in a starting dose range of 14.6$17.4 \mathrm{mg} / \mathrm{kg}$. Once stable blood levels were reached, final doses needed to maintain blood levels at $400-600 \mathrm{ng} / \mathrm{dl}$ ranged from 12.1 to $40.0 \mathrm{mg} / \mathrm{kg}$ daily. 
and extending from the prostatic capsule displayed clear delineation between the tumor and periprostatic fat sonographically (Fig. 3A). These capsular tumors were likely due to leakage of Ace- 1 cells from primary injection sites and formed tumors with thick capsules of granulation tissue. These tumors continued to grow rapidly until the dogs were euthanized at 4 weeks post-tumor cell injection. In some instances, injection of tumor cells was followed by injection with agarose in an attempt to "plug" the injection sites and retain tumor cells within the prostate. The addition of agarose did not appear to have any impact on tumor location (data not shown).

To encourage tumor growth within the prostate parenchyma rather than in the subcapsular area, we refined our injection technique for the remaining 10 dogs by dividing the same volume of tumor cell suspension between five separate incremental injections as outlined above. In addition, we also reduced the cell number injected by half to slow tumor development to a moderate rate to facilitate tumor monitoring. Subsequent to modification of the injection technique, $60 \%$ of the dogs injected developed Ace-1 tumors within the prostate parenchyma, while the
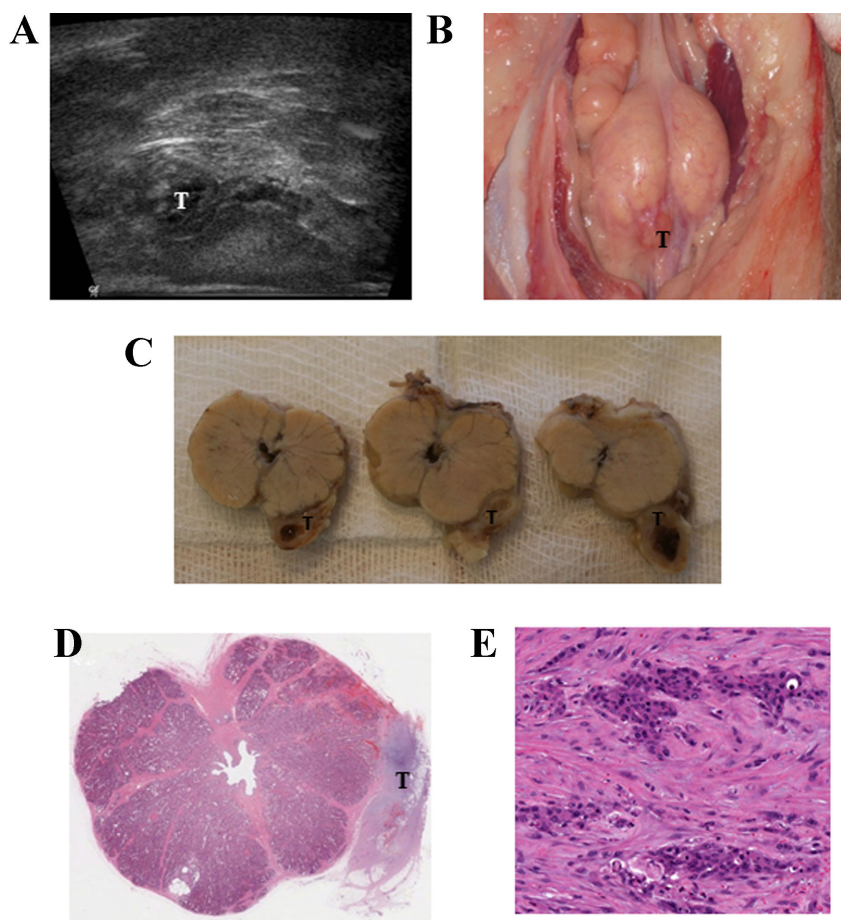

Fig. 3. Appearance of subcapsular Ace-I prostate cancer in dogs. A: Transrectal ultrasound image of subcapsular tumor $(T)$. B: In situ subcapsular tumor ( $T$ ). C: Formalin-fixed sections of subcapsular tumor (T). D: Histology of the entire prostate gland with subcapsular tumor $(\mathrm{T})$ showing multinodular, invasive, prostate carcinoma growing in prostate capsule and adjacent connective tissue and skeletal muscle. E: Magnified appearance of subcapsular tumor showing desmoplasia and marked central necrosis.

other $40 \%$ developed tumors within the subcapsular area of the prostate. On transrectal ultrasound, intraprostatic tumors appeared as discrete hypoechoic lesions within the adjacent normal appearing parenchyma (Fig. 4A). In some dogs, prostate volume increased over time (Fig. 4B); however, this was not readily apparent in all dogs.

Histopathological analysis revealed moderate cystic or papillary benign prostatic hyperplasia in all dogs. Most dogs had mild to moderate lymphoplasmacytic prostatitis. Tumors were limited to the lateral prostatic lobe that received the injection of tumor cells. Tumors that grew in the prostate parenchyma formed poorly demarcated masses that invaded into the prostatic glands, ducts, and interlobular septae (Fig. 5). Tumor nodules often had central necrosis. The prostate carcinomas induced mild desmoplasia. In some cases, there was invasion of Ace- 1 cells into and around vessels or nerves. Overall, the Ace-1 cells formed solid, poorly differentiated carcinomas with epithelial to mesenchymal cell transformation and an alveolar or cribiform growth pattern. The prostate carcinoma cells were large and polygonal with some cells mildly spindle-shaped. The cytoplasm was darkly stained and relatively basophilic. Nuclei were round to oval with prominent central nucleoli and occasional mitoses. As a small percentage of human prostate cancers demonstrate neuroendocrine features, we had evaluated for these properties in Ace-1 xenografts. We did not identify any neuroendocrine features in Ace- 1 tumors and they are Chromogranin A negative (data not shown).

A

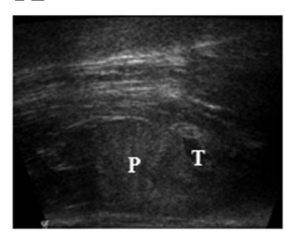

Week 1

B

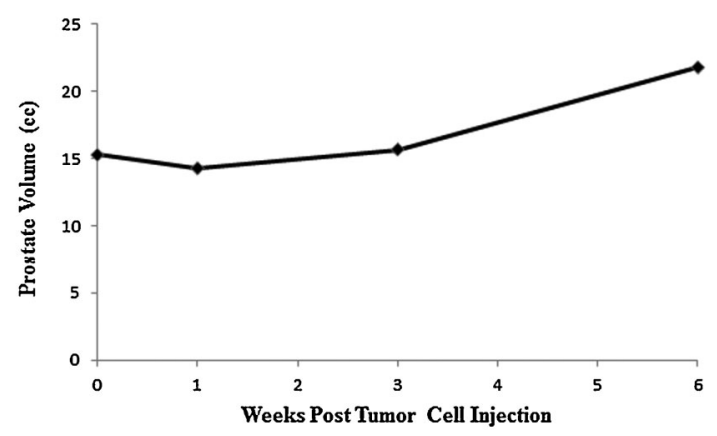

Fig. 4. Progressive growth of Ace-I tumors in dog prostate. A: Transrectal ultrasound images in the same subject over time demonstrating tumor growth. Tumor (T). Normal prostate (P). B: Prostate volume over time in the same dog. 
A

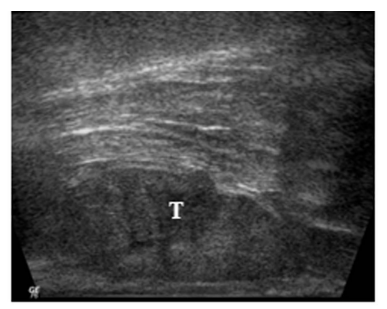

C
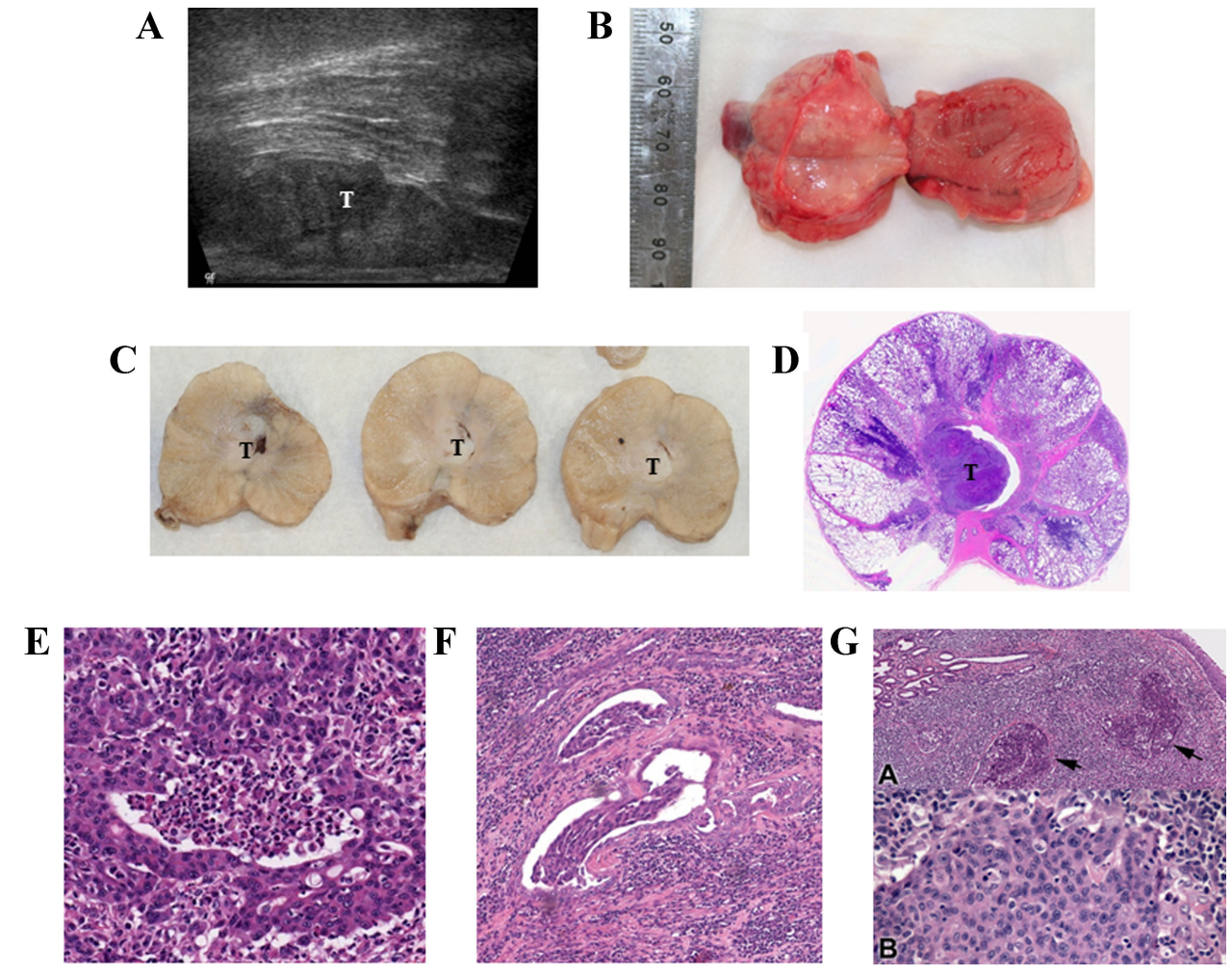

Fig. 5. Appearance of intraprostatic Ace-I tumor in dog following modifications in injection technique. A: Transrectal ultrasound image of intraprostatic tumor (T). B: Gross appearance of prostate containing intraprostatic tumor. C: Formalin-fixed sections of intraprostatic tumor (T). D: Histology of the entire prostate gland with an intraprostatic tumor ( $T$ ). Prostate carcinoma invaded into prostatic glands, ducts, and interlobular septae. E: Histologic appearance of tumor showing central necrosis and desmoplasia. F: Lymphovascular invasion of Ace-I prostate cancer cells. G: Upper panel (A) demonstrates two foci of Ace-I canine prostate cancer (arrows) growing adjacent to the urethra (upper right). Normal glands are located in the upper left. The foci of prostate cancer are in a region of chronic lymphoplasmacytic prostatitis. Lower panel (B) shows a higher magnification of the Ace-I prostate cancer cells growing in solid pattern.

Metastases to distant sites, including lungs and regional lymph nodes, occurred in 50\% of dogs following technique refinement (Fig. 6 and Table I). The majority of the metastases $(80 \%)$ occurred in dogs with intraprostatic tumors, while $20 \%(\mathrm{n}=1)$ occurred in a dog with a subcapsular tumor. Metastatic tumors formed in regional lymph nodes, lungs, and adjacent fascia. Metastases in the lungs formed nodules adjacent to bronchioles and often had lymphoplasmacytic inflammation (Fig. 6A). Metastases in regional lymph nodes formed discrete nodules in the cortex often with central necrosis (Fig. 6B). Circulating Ace- 1 tumor cells were not detected by CTC assay (data not shown). We were able to confirm that the assay was working properly by "spiking" some canine serum samples with human prostate cancer cells (PC-3), which served as a positive control. Ace-1 tumor cells did establish metastases at distant sites, indicating that these canine tumor cells were in circulation, but not detectable by CTC assay using the available human reagents.

In order to determine if the tumors could be imaged, we subjected two dogs to PET. Use of 11C- choline allowed detection of subcapsular tumor in the abdominal wall, but not the primary prostate tumor. In contrast, use of 11C-methionine allowed for detection of both tumor tissue in the prostate and subcapsular tumor (Fig. 7).

\section{DISCUSSION}

A wide variety of animal models for prostate cancer exist [11,12]. The majority of those animal models have been developed in rodents, especially mice, including xenograft and genetically engineered mouse models (GEMMs) [2]. None of the mouse models fully recapitulate the features of prostate cancer as it occurs in humans. While these models are well established, readily available, and relatively economical, they represent induced rather than spontaneous disease, making them less biologically relevant than a model such as the dog in which prostate cancer develops spontaneously with age as it does in humans [4]. Dogs are more genetically heterogeneous than most mouse models which is representative of what occurs in the human population. Intact male dogs are the only 
A
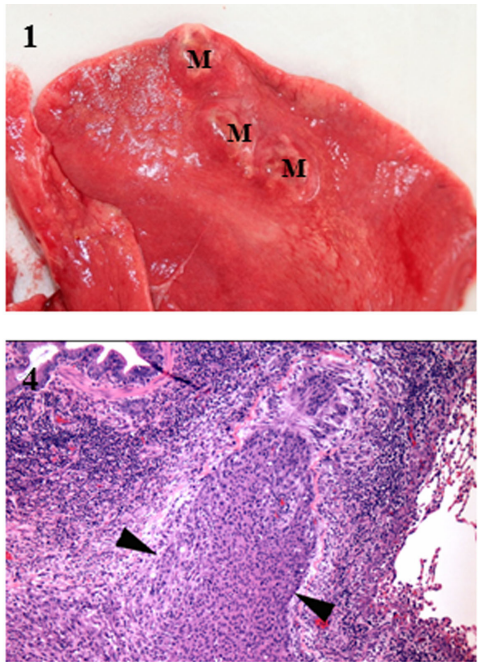

B

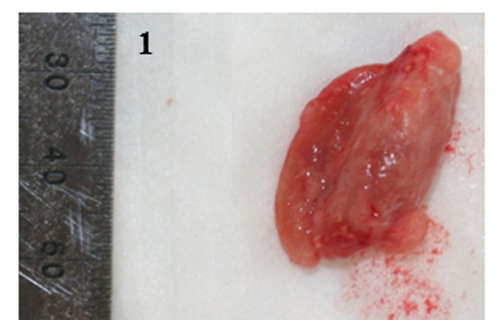

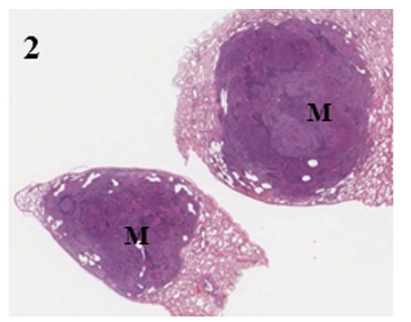
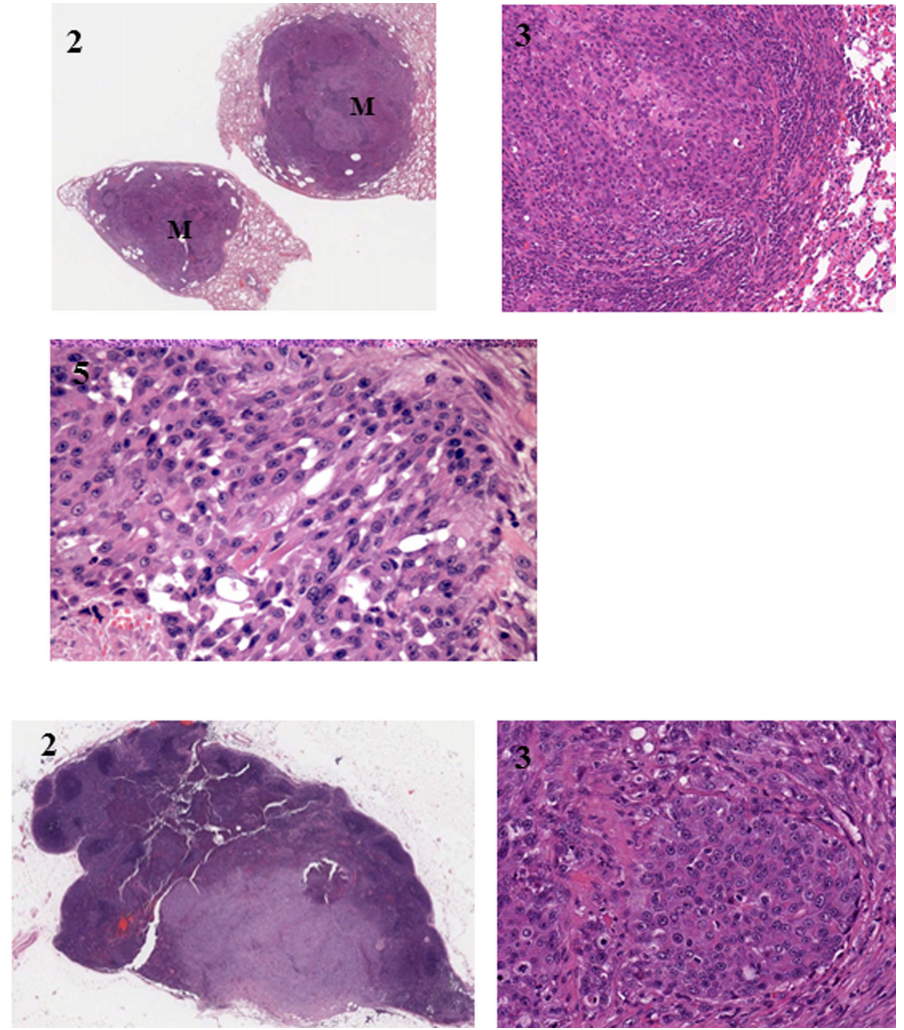

Fig. 6. Gross and histologic appearance of metastases in dogs injected orthotopically with Ace-I prostate cancer cells. A: Lung metastases. Panel I, Gross appearance of lung metastases (M). Panel 2, Histologic appearance of lung metastases. Panel 3, magnified histologic appearance. The metastasis may have originated in a peribronchiolar ar tery. It is invasive into the pulmonary parenchyma and is surrounded by a rim of lymphoplasmacytic inflammation. Panel 4, Lung metastases growing in a peribronchial vessel with invasion into the pulmonary parenchyma. A bronchiole is present in the upper left. The region has moderate lymphoplasmacytic inflammation. Alveoli are present on the right. Panel 5 , Higher magnification of Ace-I cells growing in solid and cribiform patterns. B: Lymph node metastases. Panel I, Gross appearance of lymph node metastases. Panel 2, Histologic appearance of lymph node metastases. Panel 3, magnified appearance of lymph node metastases. There is widespread, marked, cortical (T-cell) atrophy. This is a focal metastatic carcinoma with epithelial to mesenchymal cell transformation, desmoplasia, multifocal necrosis and apoptosis, and a high mitotic index.

large mammal besides humans that commonly and spontaneously develop benign prostatic hyperplasia (BPH) [13], high-grade prostatic intraepithelial neoplasia, and invasive prostate cancer with age [6,14]. This occurs independent of dog breed. There are rat strains, such as Lobund-Wistar $[15,16]$ and ACI/Seg [7], which uncommonly develop spontaneous prostate cancer, but the tumor phenotype is different and metastases do not occur as seen in men with prostate cancer [17]. In addition to the biologic relevance of spontaneous disease in the dog, there is the advantage of their larger body size compared to rodents, which allows diagnostic and surgical procedures that are used in human clinical practice. The dog prostate also shares anatomic and functional similarities with humans, including the presence of a circumferential bi-lobed single gland (compared with 4 in mice and rats) [18]. Dogs with prostate cancer develop distant metastases, including bone, which shows mixed osteoblastic and osteolytic lesions with the formation of

TABLE I. Tumor Incidence and Metastasis in Dogs Injected Orthotopically With Multiple boluses Of Ace-I Prostate Cancer Cells

\begin{tabular}{lccccc}
\hline Tumor take & Tumor in capsule & Tumor in prostate & Lung Mets & LN Mets & All Mets \\
\hline $10 / 10$ & $4 / 10$ & $6 / 10$ & $4 / 10$ & $5 / 10$ & $5 / 10$ \\
\hline
\end{tabular}



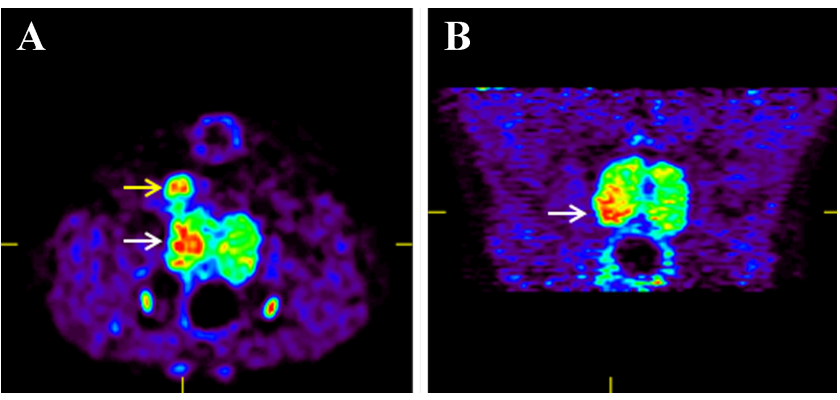

Fig. 7. Transaxial (A) and coronal (B) "C-methionine PET images of an untreated control dog injected with Ace-I prostate cancer cells. Images were taken prior to euthanasia at 6 weeks post-injection. White arrows $=$ intraprostatic tumor. Yellow arrow $=$ extracapsuracapsular tumor.

new woven bone, as seen in humans [7]. Rodents rarely develop bone metastases, and when they do they are typically osteolytic [17].

Similar to human prostate cancer cells, Ace- 1 cells express factors such as parathyroid hormone-related protein (PTHrP), cathepsin $\mathrm{K}$, keratins $8 / 18$, and vimentin [8]. PTHrP has been implicated in the development of bone metastases of human prostate cancer [19]. Cathepsin $\mathrm{K}$ has been suggested to play a role in prostate cancer progression to bone in men [20]. Expression of keratins and vimentin in human cancers may indicate that the cancer is undergoing epithelial to mesenchymal transition (EMT) [21], a feature which is observed in tumors derived from the Ace-1 cell line. Ace- 1 cells are androgen receptor (AR) negative, as are most canine prostate cancers. This may conflict with many human prostate cancers which can have normal or increased AR expression although heterogeneous AR expression is often reported [22]. Another difference among human and canine prostate cancers is the observation that many canine prostate cancers can form in castrated dogs [23]. In contrast, prostate cancer forms in men with intact testes. Thus, these differences in prostate pathophysiology should be considered upon using this model.

Despite the advantages of the dog model, as with all animal models, there are some disadvantages. Dogs are more expensive to maintain and large animal housing space must be available. It can also be difficult to obtain older, intact male dogs necessary for these studies, though dog suppliers may be able to provide retired breeders. In addition, prostate cancer in dogs is typically late-stage and androgen-independent. In contrast, prostate cancer in men is initially androgen-dependent and responsive to androgen ablation therapy and later progresses to androgen independence $[4,24,25]$. Despite these shortcomings, the advantages of the dog as a model for prostate cancer outweigh its potential disadvantages, especially in being able to provide a large animal model of prostate cancer. More specifically, canine prostate cancer is potentially a good model of late stage, androgen-independent prostate cancer with metastasis to bone, lymph nodes, and lung.

A similar dog model for prostate cancer was recently described [26]. This study was able to establish orthotopic prostate cancer in dogs immunosuppressed with cyclosporine using the DPC-1 canine cell line. Similar to our findings, tumors developed readily in the prostate and metastasis to lung and lymph nodes was detected. However, in that study, they established tumors through an invasive laparotomy; whereas, we established tumors in a less-invasive manner by using transabdominal percutaneous injection under ultrasound guidance. We also optimized our cyclosporine dosages for each individual dog to maintain consistent blood levels across subjects both prior to and after tumor cell injection. Furthermore, we were able to monitor tumors by transrectal ultrasound rather than transabdominal sonography, which is more clinically relevant. We were also able to detect both primary tumor and metastases via PET imaging. Identifying precise tumor boundaries and distinguishing tumor from normal surrounding prostate parenchyma can be challenging using any form of ultrasound, thus the use of PET imaging can more accurately differentiate the two. Both studies ultimately demonstrate the utility and feasibility of a dog model for prostate cancer.

\section{CONCLUSIONS}

In conclusion, we have successfully established a minimally invasive dog model of prostate cancer. Our model consistently developed tumors following orthotopic injection of syngeneic prostate cancer cells, and modifications in our technique led to improvements in localization of these tumors to the prostatic parenchyma. Few to no side effects were observed due to immunosuppression with cyclosporine, though the rapidity with which these tumors developed leads us to question whether these tumors may be able to grow either without or with decreased immunosuppression in future studies. We also observed metastases in approximately half of our dogs, indicating the utility of this model for studying prostate cancer progression and distant metastasis.

The orthotopic Ace-1 dog model of prostate cancer has great potential for advancing studies in this field. Potential areas of investigation include chemotherapy, surgical therapy, local ablative therapy, studies on growth, local invasion, and metastasis, bone metastasis studies on bone formation and resorption, and investigations on tumor microenvironment in a host that naturally develops this disease. 


\section{REFERENCES}

1. Jemal A, Bray F, Center MM, Ferlay J, Ward E, Forman D. Global cancer statistics. CA Cancer J Clin 2011;61(2):69-90.

2. Wang F. Modeling human prostate cancer in genetically engineered mice. Prog Mol Biol Transl Sci 2011;100:1-49.

3. Hensley PJ, Kyprianou N. Modeling prostate cancer in mice: Limitations and opportunities. J Androl 2012;33(2):133-144.

4. Leroy BE, Northrup N. Prostate cancer in dogs: Comparative and clinical aspects. Vet J 2009;180(2):149-162.

5. Coffey DS, Walsh PC. Clinical and experimental studies of benign prostatic hyperplasia. Urol Clin North Am 1990;17(3): 461-475.

6. Waters DJ, Bostwick DG. The canine prostate is a spontaneous model of intraepithelial neoplasia and prostate cancer progression. Anticancer Res 1997;17(3A):1467-1470.

7. Rosol TJ, Tannehill-Gregg SH, LeRoy BE, Mandl S, Contag CH. Animal models of bone metastasis. Cancer 2003;97(3 Suppl): 748-757.

8. LeRoy BE, Thudi NK, Nadella MV, Toribio RE, TannehillGregg SH, van Bokhoven A, Davis D, Corn S, Rosol TJ. New bone formation and osteolysis by a metastatic, highly invasive canine prostate carcinoma xenograft. Prostate 2006;66(11): 1213-1222.

9. Thudi NK, Martin CK, Murahari S, Shu ST, Lanigan LG, Werbeck JL, Keller ET, McCauley LK, Pinzone JJ, Rosol TJ. Dickkopf-1 (DKK-1) stimulated prostate cancer growth and metastasis and inhibited bone formation in osteoblastic bone metastases. Prostate 2011;71(6):615-625.

10. Thudi NK, Martin CK, Nadella MV, Fernandez SA, Werbeck JL, Pinzone JJ, Rosol TJ. Zoledronic acid decreased osteolysis but not bone metastasis in a nude mouse model of canine prostate cancer with mixed bone lesions. Prostate 2008;68(10): 1116-1125.

11. Pienta KJ, Abate-Shen C, Agus DB, Attar RM, Chung LW, Greenberg NM, Hahn WC, Isaacs JT, Navone NM, Peehl DM, Simons JW, Solit DB, Soule HR, VanDyke TA, Weber MJ, Wu L, Vessella RL. The current state of preclinical prostate cancer animal models. Prostate 2008;68(6):629-639.

12. Russell PJ, Voeks DJ. Animal models of prostate cancer. Methods Mol Med 2003;81:89-112.
13. Mahapokai W, Van Sluijs FJ, Schalken JA. Models for studying benign prostatic hyperplasia. Prostate Cancer Prostatic Dis 2000;3(1):28-33.

14. Madewell BR, Gandour-Edwards R, DeVere White RW. Canine prostatic intraepithelial neoplasia: Is the comparative model relevant? Prostate 2004;58(3):314-317.

15. Pollard M, Suckow MA. Hormone-refractory prostate cancer in the Lobund-Wistar rat. Exp Biol Med (Maywood) 2005;230(8): 520-526.

16. Pollard M. The Lobund-Wistar rat model of prostate cancer. J Cell Biochem Suppl 1992;16H:84-88.

17. Blouin S, Basle MF, Chappard D. Rat models of bone metastases. Clin Exp Metastasis 2005;22(8):605-614.

18. Evans HE. Miller's anatomy of the dog, 3rd edition. Philadelphia: W.B. Saunders; 1993.

19. Park SI, McCauley LK. Nuclear localization of parathyroid hormone-related peptide confers resistance to anoikis in prostate cancer cells. Endocr Relat Cancer 2012;19(3):243-254.

20. Podgorski I, Linebaugh BE, Sloane BF. Cathepsin K in the bone microenvironment: Link between obesity and prostate cancer? Biochem Soc Trans 2007;35(Pt 4):701-703.

21. Thompson EW, Newgreen DF, Tarin D. Carcinoma invasion and metastasis: A role for epithelial-mesenchymal transition? Cancer Res 2005;65(14):5991-5995; discussion 5995.

22. Avila DM, Zoppi S, McPhaul MJ. The androgen receptor (AR) in syndromes of androgen insensitivity and in prostate cancer. J Steroid Biochem Mol Biol 2001;76(1-5):135-142.

23. Teske E, Naan EC, van Dijk EM, Van Garderen E, Schalken JA. Canine prostate carcinoma: Epidemiological evidence of an increased risk in castrated dogs. Mol Cell Endocrinol 2002; 197(1-2):251-255.

24. Argyle DJ. Prostate cancer in dogs and men: A unique opportunity to study the disease. Vet J 2009;180(2):137-138.

25. Fork MA, Murua Escobar H, Soller JT, Sterenczak KA, Willenbrock S, Winkler S, Dorsch M, Reimann-Berg N, Hedrich HJ, Bullerdiek J, Nolte I. Establishing an in vivo model of canine prostate carcinoma using the new cell line CT1258. BMC Cancer 2008;8:240.

26. Anidjar M, Scarlata E, Cury FL, Rocha J, Hamel L, Luz M, Chevalier S. Refining the orthotopic dog prostate cancer (DPC)-1 model to better bridge the gap between rodents and men. Prostate 2012;72(7):752-761. 